\title{
Teorias da conspiração: significados em contexto brasileiro
}

\author{
Conspiracy theories: Meanings in \\ the Brazilian context
}

\author{
Alessandro Teixeira REZENDE ${ }^{1}$ (iD) 0000-0002-5381-2155 \\ Flávia Marcelly de Sousa Mendes da SILVA ${ }^{1}$ ID 0000-0001-8834-9466 \\ Maria Gabriela Costa RIBEIRO1 ${ }^{1}$ iD 0000-0001-6920-9070 \\ Gleidson Diego Lopes LOURETO' ${ }^{1}$ iD 0000-0002-0889-6097 \\ Olindina Fernandes da SILVA NETA ${ }^{1}$ iD 0000-0002-5785-3536 \\ Valdiney Veloso GOUVEIA ${ }^{1}$ iD 0000-0003-2107-5848
}

\section{Resumo}

O presente estudo teve como objetivo conhecer os significados atribuídos às teorias da conspiração em amostra brasileira. Participaram 383 estudantes universitários $\left(54,6 \%\right.$ mulheres; $\left.M_{\text {idade }}=21,9\right)$ de uma instituição pública de João Pessoa, Paraíba, que responderam a perguntas demográficas e a duas questões abertas ( $O$ que você entende por teorias da conspiração? O que Ihe vem à cabeça quando você pensa em teorias da conspiração?). A partir da análise de Classificação Hierárquica Descendente, identificaram-se cinco classes: (1) teorias sem embasamento científico, (2) manipulação de grupos secretos, (3) explicação da realidade social, (4) contestação de fatos sociais e (5) controle de informação. No geral, os significados atribuídos às teorias da conspiração foram atrelados à explicação de acontecimentos da realidade social e à existência de grupos secretos que controlam eventos importantes. Tais resultados foram discutidos à luz de estudos sobre as teorias da conspiração em Psicologia, e revelaram congruência com a concepção que se construiu desse construto.

Palavras-chave: Crenças; Significado; Teorias.

$\nabla \nabla \nabla$

1 Universidade Federal da Paraíba, Centro de Ciências Humanas, Letras e Artes, Programa de Pós-Graduação em Psicologia. Cidade Universitária, Campus I, s/n., Castelo Branco III, 58051-085, João Pessoa, PB, Brasil. Correspondência para/Correspondence to: A.T. REZENDE. E-mail: <alessandro.teixeira.rezende@gmail.com>.

Artigo elaborado a partir da dissertação de A.T. REZENDE, intitulada "Entendendo as teorias da conspiração: contribuição dos traços de personalidade e valores humanos". Universidade Federal da Paraíba, 2018.

\section{$\boldsymbol{\nabla} \boldsymbol{\nabla} \boldsymbol{v}$}

Como citar este artigo/How to cite this article

Rezende, A. T., Silva, F. M. S. M., Ribeiro, M. G. C., Loureto, G. D. L., Silva Neta, O. F., \& Gouveia, V. V. (2019). Teorias da conspiração: significados em contexto brasileiro. Estudos de Psicologia (Campinas), 36, el80010. http://dx.doi.org/10.1590/1982-02 75201936e180010 


\begin{abstract}
The aim of the study was to understand the meanings attributed to conspiracy theories in a Brazilian sample. Participants were 383 undergraduate students (54.6\% females; $\left.M_{\text {age }}=21.9\right)$ from a public institution in João Pessoa, Paraiba. They answered demographic questions and two open-ended questions (What do you understand by conspiracy theories?; What comes to your mind when you think of conspiracy theories?). Based on the descending hierarchical classification analysis, five classes were identified: (1) theories without scientific foundation, (2) manipulation of secret groups, (3) explanation of social reality, (4) contestation of social facts, and (5) information control. In general, the meanings attributed to conspiracy theories were associated with the explanation of events in social reality and existence of secret groups that control important events. These findings were discussed in light of studies on conspiracy theories in Psychology, and they revealed congruence with the conception that was developed for this construct.
\end{abstract}

Keywords: Beliefs; Meaning; Theories.

Na sociedade contemporânea as pessoas são frequentemente confrontadas com eventos que ameaçam a ordem social, tais como ataques terroristas, guerras e crises econômicas. Tais eventos, por vezes, dão origem a teorias da conspiração, que podem ser definidas como crenças explicativas utilizadas para compreender as ações de grupos ou organizações que se unem em um acordo secreto e tentam atingir um objetivo oculto, sendo este percebido como ilegal ou malévolo (Barron, Morgan, Towell, Altemeyer, \& Swami, 2014).

A internet, por exemplo, reúne vários casos de tais teorias da conspiração. Por exemplo, sugere-se que os ataques terroristas de 11 de setembro foram conduzidos pelo governo do ex-presidente estadunidense George Bush e que a guerra do Iraque foi resultado de um plano secreto promovido por empresas poderosas do setor petrolífero. É nesse sentido que os termos "conspiração", "conspiracionismo", ou, ainda, "pensamento conspiratório" são disseminados no cotidiano e fazem parte do vocabulário dos indivíduos, sobretudo no contexto das redes sociais (Bessi et al., 2015).

Nessa conjuntura, as teorias da conspiração vão estar associadas à criação de uma explicação "alternativa" ou "fantasiosa" para fatos que normalmente contrariam a versão oficial e politicamente correta de um determinado acontecimento (Van Prooijen \& Acker, 2015). Os criadores e adeptos de tais teorias, em geral, apresentam um olhar cético frente a algumas explicações dadas a eventos controlados por instituições e pessoas poderosas, por vezes atribuindo um significado interpretativo diferente ao acontecimento (Brotherton \& Eser, 2015), comumente sugerindo explicações pouco sustentáveis moralmente.

Uma das principais características das teorias da conspiração diz respeito a fornecerem explicações causais para eventos sociais complexos. A propósito, Hofstadter (1966) indica que a ideia conspiradora está enraizada em uma tendência geral de explicar e racionalizar fenômenos complexos do mundo real em um conjunto coerente de pressupostos sobre a existência de um inimigo poderoso e malvado, e ademais, destaca a necessidade de as pessoas explicarem eventos que são difíceis de compreender. Isso sugere que a crença em teorias da conspiração reflete um método sistemático de processamento de informação, que enseja uma visão de mundo geral capaz de explicar eventos tidos como ameaçadores ou desconhecidos (Jolley, Douglas, \& Sutton, 2017; Leman \& Cinnirella, 2013).

Durante muito tempo as teorias da conspiração foram deixadas, quase que completamente, à mercê modelo explicativo leigo, diversos psicólogos passaram a dar atenção ao fenômeno. A propósito, no final dos anos 1980, Carl Graumann observou que, embora as teorias da conspiração fossem consideradas um tópico de "interesse psicológico intrínseco", não havia a presença de um corpo substancial de pesquisas psicológicas dedicadas especificamente a essa temática (Van Prooijen \& Acker, 2015). Nessa conjuntura, diversos estudos começaram a explorar os fatores psicológicos que poderiam explicar a suscetibilidade que as pessoas apresentavam a crenças conspiratórias, e promoveram, assim, um gradual surgimento no interesse da Psicologia em estudar essa temática (Byford, 2014). 
Há alguns anos as teorias da conspiração estiveram atreladas a visões políticas extremistas e, por conseguinte, deixaram de lado a importância que poderiam ter na explicação de eventos sociais. No entanto, nas duas últimas décadas houve uma transformação importante na percepção pública dessas teorias (Lobato, Mendoza, Sims, \& Chin, 2014). Especificamente, as ideias ou crenças conspiratórias passaram a ser vistas não mais como uma simples prerrogativa de extremistas, mas como uma forma de explicação social cotidiana e uma maneira cada vez mais comum de compreender questões diversas, como o contexto político, o aumento da vigilância, a ameaça à privacidade proveniente do desenvolvimento da tecnologia ou o aumento do poder das corporações transnacionais (Jolley \& Douglas, 2014a). Seguindo essa linha de pensamento, as teorias da conspiração passaram a ser vistas como uma forma de dar sentido à incerteza cognitiva do cotidiano. Ao serem confrontadas com informações sobre um evento considerado inexplicável, as pessoas procuram minimizar a incerteza por meio de "atalhos cognitivos", que fazem com que os indivíduos sigam a lógica de ideias conspiratórias (Jolley et al., 2017).

A relevância das teorias da conspiração é sustentada por sua recente inserção no campo de pesquisa da Psicologia (Jolley et al., 2017; Lobato et al., 2014; Oliver \& Wood, 2014; Swami, Voracek, Stieger, Tran, \& Furnham, 2014) que, em síntese, dá enfoque na identificação de fatores que distinguem os indivíduos que endossam crenças conspiratórias daqueles que não o fazem. Além disso, as pesquisas nessa área têm se centrado em identificar os deficits perceptuais ou cognitivos que levam os indivíduos a adotar explicações conspiratórias. Nessa direção, de acordo com Kruglanski (1987), as teorias da conspiração podem ser sustentadas por ilusões cognitivas capazes de dar coerência a uma realidade não explicável. Parece mesmo inegável a influência que as teorias da conspiração exercem no cotidiano dos indivíduos; por meio de uma pesquisa de opinião realizada por Byford (2011), por exemplo, verificou-se que uma proporção substancial (cerca de 90\%) de pessoas no mundo ocidental admite acreditar em alguma forma de teoria conspiratória.

Por serem disseminadas na sociedade, as teorias em questão vão ocasionar consequências sociais tanto positivas quanto negativas na vida dos indivíduos. Por exemplo, embora a crença em teorias da conspiração possa promover uma maior transparência política (Swami \& Coles, 2010) e contestação das estruturas ideológicas dominantes (Sapountzis \& Condor, 2013), há evidências de que a exposição a tais teorias leva a uma redução na intenção de se envolver em políticas voltadas à redução de monóxido de carbono (Jolley \& Douglas, 2014a), nas atitudes positivas em relação a vacinas (Jolley \& Douglas, 2014b) e no engajamento em comportamentos positivos no âmbito da saúde (Oliver \& Wood, 2014). Bogart e Thorburn (2006), por exemplo, mostraram que a exposição de teorias da conspiração entre os afro-americanos esteve associada a atitudes negativas em relação a comportamentos contraceptivos, e gerou consequências negativas quanto à prevenção de gravidez e incidência de doenças sexualmente transmissíveis.

Apesar de serem identificados estudos sobre a temática no contexto internacional no campo da Psicologia (Oliver \& Wood, 2014; Sapountzis \& Condor, 2013), sobretudo investigações de cunho quantitativo e experimental (Lobato et al., 2014; Moulding et al., 2016), ainda são escassas as pesquisas no Brasil que se dedicam a estudar esse fenômeno. Buscas realizadas em bases de dados brasileiras em 13 de janeiro de 2018 (IndexPsi, PePSIC, SciELO e Lilacs), com o uso dos descritores "teorias", "conspiração" e "Psicologia", não encontraram nenhum estudo empírico que tivesse sido publicado nos últimos cinco anos. Ao ampliar a busca ao Google Acadêmico, empregando-se os mesmos descritores, foram localizados 4.370 registros. Porém, esses registros não contemplavam a Psicologia, mas áreas conexas (Ciências Sociais, Comunicação, História), que frequentemente abordavam o tema de forma teórica ou discursiva (Nícolas, 2017; Trifonova, 2017).

A partir do anteriormente descrito e tendo em conta a centralidade que as teorias conspiratórias assumem na vida das pessoas, parece justificável realizar esforços para entender os significados que estas atribuem ao construto. Desse modo, o presente estudo teve como objetivo conhecer os significados que as pessoas associam às teorias da conspiração. Tal empreendimento apresenta relevância social, tendo em vista que essas teorias persistem como um meio popular de articular oposição às forças do capitalismo internacional 
e da globalização (Byford, 2011), possibilitando o enfrentamento às hierarquias sociais estabelecidas e oferecendo entendimentos alternativos sobre eventos da realidade social (Sapountzis \& Condor, 2013).

\section{Método}

\section{Participantes}

Participaram deste estudo 383 estudantes universitários de uma instituição pública de João Pessoa (PB), cujas idades variaram entre 18 e 54 anos $(M=21,9 ; D P=5,07)$, sendo a maioria do sexo feminino (54,6\%), solteira (93,8\%), católica $(40,8 \%$ ) e heterossexual (84,8\%). Tratou-se de uma amostra de conveniência (não probabilística), composta por pessoas que, solicitadas a colaborar, aceitaram fazê-lo voluntariamente.

\section{Instrumentos}

Os participantes foram solicitados a preencher um questionário com uma única página que reunia perguntas de caráter demográfico para caracterização da amostra (idade, sexo, estado civil, orientação sexual e religião) e duas questões abertas que indagavam (1) o que a pessoa entendia por teorias da conspiração e (2) o que the vinha à cabeça quando ela pensava em teorias da conspiração.

\section{Procedimentos}

A coleta de dados foi realizada por dois colaboradores devidamente treinados. Esta teve lugar em ambiente coletivo de sala de aula, após autorização dos professores responsáveis pelas turmas, sendo as respostas dadas individualmente. Garantiu-se o caráter anônimo e a natureza voluntária da participação, bem como foi assegurado o direito de desistência sem quaisquer consequências aos participantes, que tiveram que assinar um Termo de Consentimento Livre e Esclarecido (TCLE), de acordo com o que estabelece a Resolução 510/16, do Conselho Nacional de Saúde (CNS). O projeto correspondente foi aprovado pelo Comitê de Ética em Pesquisa da Universidade Federal da Paraíba (UFPB) com seres humanos (CAAE n 76972917.8.0000.5188). Em média, os participantes levaram dez minutos para completar a tarefa.

\section{Organização do banco e análise dos dados}

Os dados textuais foram transcritos no programa OpenOffice Writer, sendo organizados em um único arquivo equivalente às duas perguntas anteriormente apresentadas, e reunidos em um único corpus. Após ajustes e organização do corpus, o arquivo foi importado para o Bloco de Notas do Windows e salvo na codificação UTF-8 para análise no Iramuteq (Ratinaud, 2009), executado com o programa R (R Development Core Team, 2012). Realizaram-se três tipos de análises: (1) Classificação pelo método de Reinert - efetuou-se uma Classificação Hierárquica Descendente (CHD), nos quais os segmentos de texto são classificados segundo seus vocábulos e seu conjunto é dividido com base na frequência das formas reduzidas, formadas a partir do radical das palavras (lemmatisation) (Camargo \& Justo, 2013); (2) Nuvem de palavras - representa graficamente e organiza as palavras de acordo com suas frequências; por ser uma análise que facilita a identificação de palavras-chave a partir do banco de dados (corpus), é utilizada em contextos que necessitam expor de maneira objetiva as informações (Ratinaud, 2009); e (3) Análise de similitude - baseia-se na teoria dos grafos e identifica as coocorrências entre as palavras, que resultam na identificação de suas conexões e 4 auxilia na visualização gráfica da estrutura do corpus (Ratinaud \& Marchand, 2012). 


\section{Resultados}

O corpus analisado foi composto por 383 unidades de contexto iniciais, representando a totalidade de participantes do estudo. Foi apresentada uma média de 18,2\% de formas (o número de formas diz respeito ao número de palavras com radicais diferentes contidos no texto) por Segmento de Texto (ST), num total de 8.130 ocorrências (totalidade de palavras contidas no corpus). Estas correspondem a 84,6\% de aproveitamento das unidades de contextos elementares de ST do corpus, o que é uma porcentagem adequada para extração do banco de dados textuais (Camargo, 2005).

Inicialmente, procedeu-se à Classificação Hierárquica Descendente (CHD), tendo sido identificadas cinco classes que aparecem no dendograma (Figura 1), no qual são apresentadas as palavras de cada classe juntamente com os valores dos respectivos qui-quadrados e a frequência de cada palavra. Ressalta-se que foram incluídas apenas as palavras cujos valores de qui-quadrados eram mais altos, sendo três vezes maior que o valor mínimo estabelecido [ $\left.\chi^{2}(1) \geq 11,52, p<0,05\right]$, a fim de trabalhar com a menor margem de erro em cada associação da palavra com sua respectiva classe.

Com o objetivo de melhor compreender o processo de divisão do conteúdo textual e da constituição das classes, o dendrograma possibilita visualizar os agrupamentos possíveis a partir da CHD. Dessa forma, é possível identificar não apenas a relação entre as classes, mas também os vocábulos mais associados com cada uma delas. No caso, ao observar o dendograma em sentido horário, é possível interpretar as classes como segue:

A Classe 2 foi denominada de "Teorias sem embasamento científico" (ST classe2 $=58$, explicando $21,5 \%$ do total). Ela representa a ideia de que as teorias da conspiração são apenas opiniões criadas pelo senso comum, utilizadas para contradizer os fatos que são divulgados por organizações governamentais. Dessa forma, são entendidas como teorias hipotéticas que a população cria para entender eventos sociais, tais como decisões tomadas pelo governo ou implementação de novas tecnologias. Essa classe pode ser exemplificada a partir de falas como "teorias que não têm um embasamento científico, que vêm do senso comum"; "são ideias ou teses que a população leiga cria a respeito de decisões governamentais sem embasamento consistente"; e "são um conjunto de hipóteses mirabolantes sem comprovação alguma e que buscam explicações complexas para os eventos".

\begin{tabular}{|c|c|c|c|c|c|c|c|c|c|c|c|c|c|c|}
\hline \multicolumn{3}{|c|}{$\begin{array}{c}\text { Classe } \mathbf{2} \\
\text { Teorias sem embasamento } \\
\text { científico }\end{array}$} & \multicolumn{3}{|c|}{$\begin{array}{c}\text { Classe } 1 \\
\text { Manipulação de grupo } \\
\text { secretos }\end{array}$} & \multicolumn{3}{|c|}{$\begin{array}{c}\text { Classe } \mathbf{3} \\
\text { Explicação da realidade } \\
\text { social }\end{array}$} & \multicolumn{3}{|c|}{$\begin{array}{c}\text { Classe } \mathbf{5} \\
\text { Contestação de fatos sociais }\end{array}$} & \multicolumn{3}{|c|}{$\begin{array}{c}\text { Classe } \mathbf{4} \\
\text { Controle de informação }\end{array}$} \\
\hline Palavra & $\mathrm{f}$ & $\chi^{2}$ & \begin{tabular}{|l|} 
Palavra \\
\end{tabular} & $\mathrm{f}$ & $\chi^{2}$ & \begin{tabular}{|l|} 
Palavra \\
\end{tabular} & $f$ & $\chi^{2}$ & Palavra & $f$ & $\chi^{2}$ & \begin{tabular}{|l|} 
Palavra \\
\end{tabular} & $f$ & $\chi^{2}$ \\
\hline $\begin{array}{l}\text { Realidade } \\
\text { Comum } \\
\text { Extraterrestre } \\
\text { Prova } \\
\text { Senso } \\
\text { Pouco } \\
\text { Sentido } \\
\text { Científico } \\
\text { Embasamento } \\
\text { Gerar } \\
\text { Comprovação } \\
\end{array}$ & $\begin{array}{c}14 \\
15 \\
8 \\
10 \\
9 \\
8 \\
11 \\
7 \\
4 \\
4 \\
4\end{array}$ & $\begin{array}{l}36,1 \\
32,2 \\
30,1 \\
28,9 \\
25,0 \\
21,3 \\
17,8 \\
17,5 \\
14,8 \\
14,8 \\
14,8\end{array}$ & \begin{tabular}{|l} 
Secreto \\
Plano \\
Acreditar \\
Sociedade \\
Manter
\end{tabular} & $\begin{array}{l}33 \\
10 \\
21 \\
23 \\
6\end{array}$ & $\begin{array}{l}42,9 \\
27,6 \\
20,7 \\
17,1 \\
13,2 \\
\end{array}$ & $\begin{array}{l}\text { Acontecer } \\
\text { Pensar } \\
\text { Conspirar } \\
\text { Forma } \\
\text { Coisa } \\
\text { Pensamento } \\
\text { Exemplo } \\
\text { Afim } \\
\text { Alguma } \\
\text { Diferente } \\
\text { Algum } \\
\text { Influenciar } \\
\text { Negativo }\end{array}$ & $\begin{array}{c}22 \\
10 \\
11 \\
26 \\
35 \\
17 \\
4 \\
4 \\
15 \\
6 \\
28 \\
9 \\
3\end{array}$ & $\begin{array}{l}40,4 \\
31,0 \\
26,6 \\
24,7 \\
23,9 \\
16,5 \\
15,8 \\
15,8 \\
15,3 \\
15,0 \\
13,0 \\
12,3 \\
11,8\end{array}$ & $\begin{array}{l}\text { Acontecimento } \\
\text { Explicação } \\
\text { Oficial } \\
\text { Forjar } \\
\text { Tentar } \\
\text { Buscar } \\
\text { Usar } \\
\text { Explicar } \\
\text { Alternativa } \\
\text { Certo }\end{array}$ & $\begin{array}{c}43 \\
17 \\
5 \\
4 \\
13 \\
10 \\
5 \\
26 \\
6 \\
28\end{array}$ & $\begin{array}{l}44,0 \\
38,3 \\
37,8 \\
30,2 \\
23,0 \\
23,0 \\
22,6 \\
19,5 \\
17,6 \\
12,3\end{array}$ & \begin{tabular}{|l} 
Governo \\
Divulgar \\
Público \\
Levar \\
Ato \\
Envolver \\
Assassinato \\
Sua \\
Mídia \\
Controlar \\
História \\
Maçonaria \\
Importante \\
Massa
\end{tabular} & $\begin{array}{c}45 \\
9 \\
13 \\
6 \\
10 \\
10 \\
7 \\
9 \\
9 \\
14 \\
4 \\
4 \\
10 \\
10\end{array}$ & $\begin{array}{l}48,1 \\
29,3 \\
27,3 \\
19,3 \\
17,7 \\
17,7 \\
14,9 \\
14,6 \\
14,6 \\
13,0 \\
12,8 \\
12,8 \\
11,0 \\
11,9\end{array}$ \\
\hline
\end{tabular}

Figura 1. Dendograma da representação dos vocábulos das classes. 
A Classe 1 recebeu o nome de "Manipulação de grupos secretos" (ST classe1 $=60$, explicando 22,2\% do total). Está relacionada à crença de que grupos anônimos possuem poder para influenciar certos acontecimentos, tais como disseminação de doenças e divulgação de informações falsas. É possível exemplificar o porquê de algumas pessoas acreditarem que as teorias da conspiração são provenientes de uma aliança secreta de organizações e indivíduos poderosos a partir de trechos como "são ideias resultantes de condições adversas que advêm de conspirações criadas por um grupo que domina o mundo", "teorias que afirmam a existência de grupos secretos que controlam a informação, tecnologia e economia para manter o mundo da forma que eles querem" e "teorias de que determinados grupos definem os acontecimentos dentro do meio social e político e que grupos secretos estão no controle das decisões da sociedade".

A Classe 3 foi denominada de "Explicação da realidade social" (ST classe3 $=55$, explicando 20,4\% do total), uma vez que se refere à propensão que as teorias conspiratórias apresentam de fornecer explicações causais para eventos sociais complexos. Dessa maneira, apresenta a ideia de que tais teorias constituem uma tendência de racionalizar e explicar eventos que são de difícil compreensão (ataques terroristas, proliferação de doenças). São exemplos de trechos que descrevem essa classe: "teorias da conspiração são teorias que tentam explicar algum evento que aconteceu e acontece, com outros argumentos ou pontos de vista deferentes do que é explicado para o resto da população; eu penso em algumas teorias como a do ataque as torres gêmeas nos EUA" e "são uma forma de explicar o porquê de a população mundial ser tão manipulada. Eu penso nas informações e acontecimentos que são ocultos do conhecimento das pessoas porque eu acho que toda a sociedade, a política e os meios de comunicação conspiram para que as pessoas se comportem dentro de um padrão".

A Classe 5 foi nomeada como "Contestação de fatos sociais" (ST classe5 $_{1}=32$, explicando 11,9\% do total). Está ligada à ideia de que teorias conspiratórias são explicações alternativas que contradizem os fatos divulgados pela mídia e o governo e estão associadas à criação de interpretações que vão contra a versão oficial e politicamente correta de um determinado acontecimento. Exemplificam essa classe respostas como "teorias que desenvolvem explicações alternativas às oficiais para determinados acontecimentos, tais como a chegada do homem à lua organizada pela CIA", "hipóteses que as pessoas criam para explicar certos acontecimentos por não acreditarem nas explicações oficiais, como os ataques do 11 de setembro e de que Michael Jackson não morreu" e "teorias que tentam dar um novo significado para fatos que já possuem uma explicação oficial e aceita pela maior parte da população".

Finalmente, a Classe 4 foi denominada "Controle de informação" (ST classe4 $=65$, explicando 24,1\% do total). Relacionada à Classe 5, refere-se ao fato de que muitas informações importantes (cura para doenças como câncer e o Vírus da Imunodeficiência Humana (HIV, Human Immunodeficiency Virus); assassinato de figuras importantes) não são repassadas para o público. Trechos que exemplificam essa classe são: "teorias que vão contra o que está estabelecido e partem do saber popular envolvendo ações que o governo promove às escondidas, como assassinatos de figuras públicas e doenças incuráveis", "atos e manobras que são realizados com o intuito de controlar a opinião das pessoas na sociedade, controlando a sociedade conforme seus interesses" e "uma forma de controlar a massa e encobrir certas informações".

Uma vez conhecidas as classes que surgiram, procurou-se uma solução gráfica capaz de representar as palavras mais frequentemente utilizadas no corpus de maneira geral. Nesse sentido, realizou-se uma análise de nuvem de palavras (Figura 2), a qual dispõe os termos em razão de suas frequências, sendo os termos mais frequentemente utilizados aqueles centrais e de tamanhos maiores.

Pode-se observar que alguns termos mais frequentes e que atuam como palavras-chave do corpus foram: secreto, governo, manipular, pessoa, teoria, fato e controle. De forma geral, essa disposição gráfica dos dados corrobora os achados anteriores expressos nas classes da CHD, no tocante à ideia da existência de grupos secretos que controlam e manipulam decisões importantes com o intuito de atingir interesses próprios. 6 Ademais, a presença destacada de vocábulos como explicar, informação, pensamento e acontecimento indica 


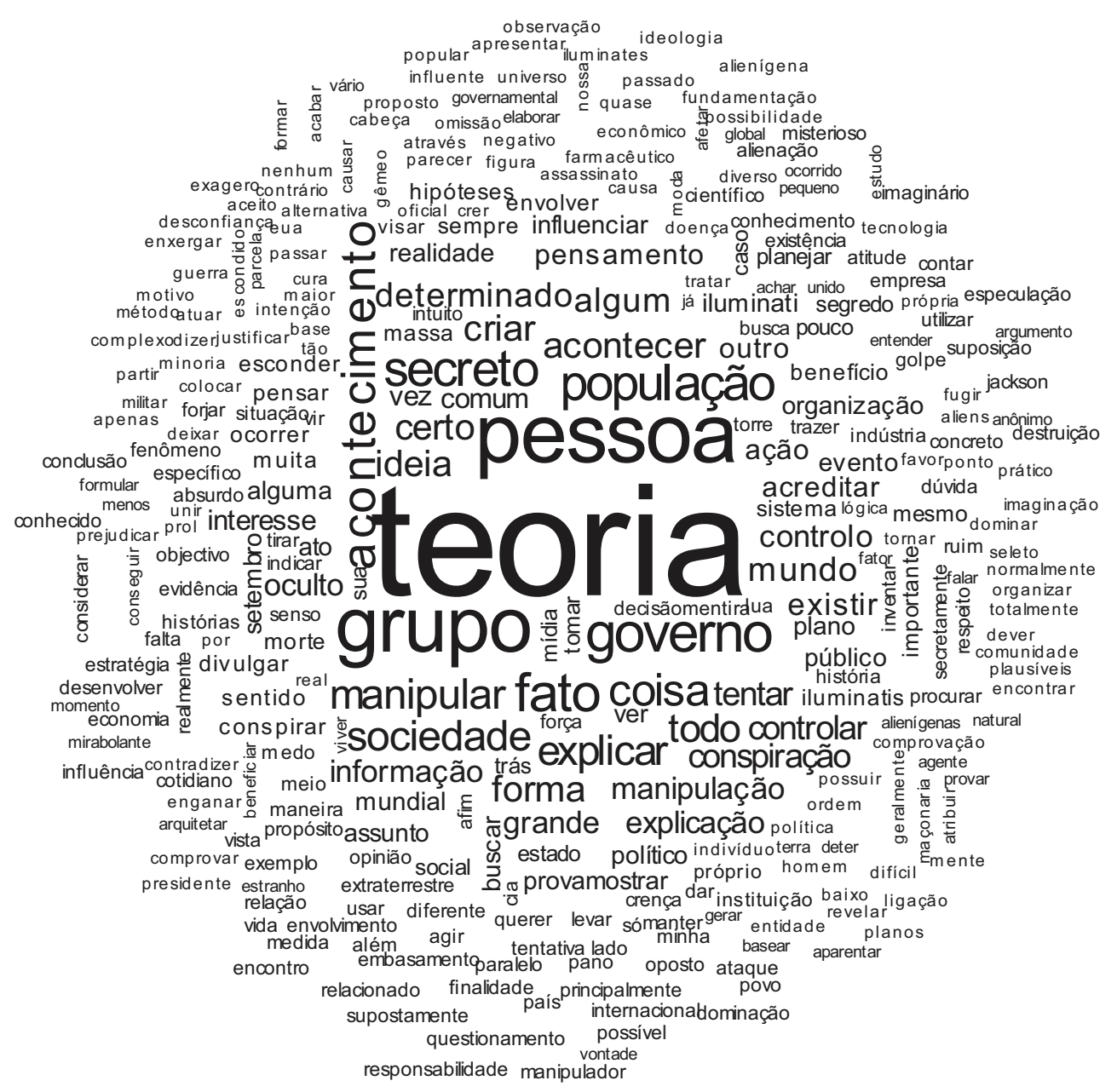

Figura 2. Nuvem de palavras.

que as teorias da conspiração são tidas como uma forma de explicar os eventos da realidade social, para os quais os indivíduos criam interpretações "alternativas", especialmente em eventos de alta complexidade, como o assassinato de pessoas importantes e a disseminação de doenças.

Por fim, no intuito de complementar a nuvem de palavras, realizou-se uma análise de similitude. Também fundamentada na frequência com que as palavras ocorrem, essa análise permite indicar a conectividade entre os vocábulos em auxílio à identificação da estrutura e representação do corpus. A Figura 3 representa a árvore de coocorrências.

É possível observar que a árvore de coocorrências é composta, principalmente, por quatro eixos centrais, formados pelos termos teoria, acontecimento, grupo e governo. Precisamente, esses eixos centrais dão origem à proposição de que as teorias da conspiração decorrem da ação de um grupo, organização ou instituição que, por meio de planos secretos, executa ações ilegais ou prejudiciais à sociedade em geral.

\section{Discussão}

Como previamente indicado, pretendeu-se no presente estudo identificar os significados atribuídos às teorias da conspiração a partir da análise das falas das pessoas. Isso pareceu justificável, pois apesar de a 


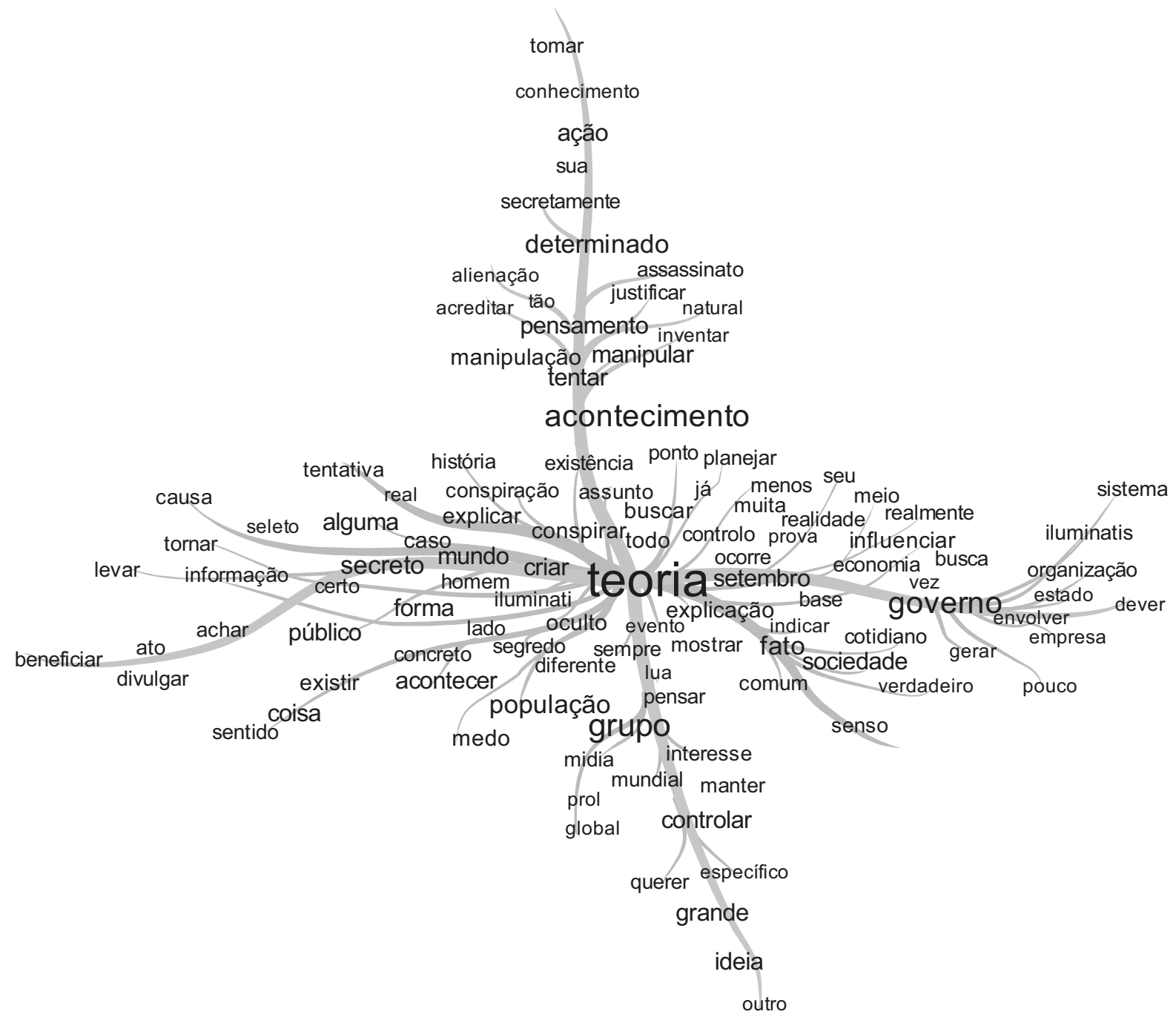

Figura 3. Árvore de coocorrências - análise de similitude.

literatura internacional reunir informações sobre essas teorias e seu impacto na vida das pessoas e organizações sociais, pouco se sabe a respeito do fenômeno no âmbito da Psicologia brasileira, mesmo que se conheça a associação das crenças nessas teorias com comportamentos prejudiciais (problemas de saúde, extremismos ideológicos, hostilidade e menor engajamento em comportamentos ambientais e políticos; Jolley \& Douglas, 2014b; Swami \& Furnham, 2012; Van Prooijen, Krouwel, \& Pollet, 2015).

A necessidade de manter controle sobre o meio social pode explicar a razão de as teorias da conspiração ganharem impulso particularmente após eventos sociais impactantes, que provavelmente são experimentados como ameaças de controle por parte dos cidadãos (ataque terrorista, guerra, desastre natural; Swami \& Furnham, 2012). Nesse cenário, a Psicologia tem como papel analítico enfatizar o motivo pelo qual as pessoas acreditam em certas ideias conspiratórias para explicar a realidade social, ao evitarem classificar as teorias da conspiração como provavelmente "verdadeiras" ou "falsas" (Douglas \& Sutton, 2008).

Os resultados previamente descritos convergiram com estudos internacionais que procuram conceituar as teorias da conspiração. Por exemplo, coerentes com o que se indica na Classe 2 (Teorias sem embasamento 8 científico), Bessi et al. (2015) destacam que as ideias conspiratórias tendem a reduzir a complexidade da 
realidade, de modo a explicar aspectos políticos ou sociais como se organizados por indivíduos ou organizações secretas. Tais explicações fazem com que as pessoas rejeitem argumentos baseados no conhecimento científico e adotem discursos alternativos para substituir evidências proporcionadas pela ciência. Desse modo, as pessoas que negam a relação entre HIV e AIDS (Acquired Immunodeficiency Syndrome), por exemplo, acreditam que esta é uma doença criada pelo governo dos Estados Unidos para contaminar a população afro-americana. Na mesma direção, Swami e Coles (2010) postulam que a adoção de crenças conspiratórias decorre da incapacidade que os indivíduos têm de exercer um julgamento crítico a partir de dados científicos.

No que concerne à Classe 1 (Manipulação de grupos secretos), observou-se maior ocorrência de palavras ligadas às expressões "secreto" e "plano", de forma congruente com o que descreve Goertzel (2010) ao afirmar que as teorias da conspiração são vistas muitas vezes como ligadas a indivíduos ou grupos poderosos e secretos que ocupam posições de autoridade. Nessa mesma direção, Drinkwater, Dagnall e Parker (2012) sugerem que as teorias contemporâneas da conspiração focam na existência de forças secretas e malignas que agem dentro de indústrias de medicamentos, multinacionais e laboratórios de pesquisa, produzindo as enfermidades.

A Classe 3 (Explicação da realidade social) reuniu palavras como "acontecer", "pensar", "conspirar" e "influenciar", suscitando a ideia de que as teorias conspiratórias constituem recursos cognitivos que atendem à necessidade de se explicarem eventos complexos e perturbadores, como a disseminação de doenças contagiosas e a morte repentina de celebridades (Wagner-Egger et al., 2011). A propósito, Knight (2008) sustenta que essas teorias decorrem da necessidade de entender a causalidade e as consequências de eventos significativos, noção que é sustentada também por Goertzel (1994). Este autor sugere que as teorias da conspiração fazem parte de um sistema de crenças que permite aos indivíduos compreender fenômenos ocorridos na realidade social, e indicam o porquê de eventos significativos ou impactantes serem frequentemente acompanhados de explicações leigas e especulativas.

A Classe 5 (Contestação de fatos sociais) abordou como principais descritores as palavras "acontecimento", "explicação", "oficial" e "forjar". Isso é coerente com o que descrevem Aaronovitch \& Langton (2010), que percebem as teorias da conspiração como frequentemente endossadas e aceitas quando não há uma explicação definitiva para um evento, ou quando a versão oficial de um acontecimento não é considerada verídica. Na mesma linha de pensamento, Van Prooijen e Acker (2015) alegam que a aprovação de tais teorias como forma de contestação de fatos sociais faz com que os indivíduos criem argumentos divergentes da versão considerada "politicamente correta".

Por fim, a Classe 4 (Controle de informação) se referiu a vocábulos como "governo", "divulgar", "público", "ato" e "envolver". Parece evidente que essa classe converge para a crença de que as teorias conspiratórias envolvem organizações ou grupos que se unem com o objetivo de controlar, influenciar as informações fornecidas pela mídia, disseminar versões convenientes de acontecimentos de difícil explicação ou que favoreçam interpretações especulativas (Moulding et al., 2016).

De maneira geral, os resultados observados (CHD, nuvem de palavras e análise de similitude) reforçam os obtidos em pesquisas internacionais realizadas na Psicologia. Essencialmente, sugerem que as teorias da conspiração podem ser compreendidas como tendo uma função explicativa e estando associadas a processos mentais que visam considerar o mundo como ordenado, compreensível e previsível (Brotherton \& Eser, 2015). Portanto, tais teorias são concebidas como um conjunto de pressupostos para explicar a existência de um "inimigo" e evidenciam a necessidade intrínseca que os indivíduos têm de explicar eventos sociais complexos (Van Prooijen \& Jostmann, 2013).

A partir da atenção que a temática tem recebido no contexto internacional, é perceptível que as teorias da conspiração se revelam como um construto importante para explicar diversas condutas sociais (confiança interpessoal, engajamento em políticas de saúde, contestação das estruturas ideológicas dominantes); (Leman 
\& Cinnirella, 2013; Oliver \& Wood, 2014; Sapountzis \& Condor, 2013). Sendo assim, justificam-se novos esforços para compreender fatores psicológicos subjacentes a esse fenômeno (Jolley et al., 2017). Desse modo, a presente pesquisa apenas buscou contribuir ao dar os primeiros passos, no intuito de sistematizar os significados que as pessoas atribuem a tais teorias no contexto brasileiro. No caso, adotou-se uma abordagem qualitativa e exploratória, o que propiciou o surgimento de significados livres de conotações negativas, e possibilitou o entendimento de como, efetivamente, as pessoas percebem essas teorias.

Por fim, apesar da relevância dos resultados ora descritos, que foram coerentes com os significados atribuídos às teorias da conspiração (explicação de eventos sociais, existência de grupos secretos, controle de informações); (Jolley \& Douglas, 2014a; Swami et al., 2014), como qualquer outro estudo, este não está isento de limitações. Por exemplo, a amostra reduzida e não-probabilística impede generalizar os resultados para além do grupo de participantes, estudantes universitários. Desse modo, parece interessante que sejam realizados estudos com outros grupos amostrais, incluindo pesquisa na internet com o uso de questionários online, divulgação do tema em fóruns e posterior análise dos discursos veiculados. Parece igualmente relevante conhecer os correlatos das crenças nas teorias da conspiração, que podem incluir engajamento político e comportamentos ambientais (Jolley \& Douglas, 2014b), ideologia (Byford, 2014), heurísticas e equilíbrio cognitivo (Leman \& Cinnirella, 2013), traços de personalidade (Swami, Weis, Lay, Barron, \& Furnham, 2016) e valores humanos (Gouveia, 2013), por exemplo. Nessa direção, talvez um passo preliminar compreenda contar com algum instrumento (escala, inventário) adequado à realidade brasileira, a fim de reunir evidências de seus parâmetros psicométricos.

\section{Colaboradores}

Todos os autores participaram na concepção, coleta e análise de dados, bem como na redação e revisão do artigo.

\section{Referências}

Aaronovitch, D., \& Langton, J. (2010). Voodoo histories: The role of the conspiracy theory in shaping modern history. New York: Riverhead Books.

Barron, D., Morgan, K., Towell, T., Altemeyer, B., \& Swami, V. (2014). Associations between schizotypy and belief in conspiracist ideation. Personality and Individual Differences, 70, 156-159. http://dx.doi.org/10.1016/j.paid.2014.06.040

Bessi, A., Coletto, M., Davidescu, G. A., Scala, A., Caldarelli, G., \& Quattrociocchi, W. (2015). Science vs conspiracy: Collective narratives in the age of misinformation. PLoS One, 10(2), 1-17. http://dx.doi.org/10.1371/journal. pone. 0118093

Bogart, L. M., \& Thorburn, S. (2006). Relationship of African Americans' sociodemographic characteristics to belief in conspiracies about HIVIAIDS and birth control. Journal of the National Medical Association, 98(7), 1144-1150.

Brotherton, R., \& Eser, S. (2015). Bored to fears: Boredom proneness, paranoia, and conspiracy theories. Personality and Individual Differences, 80, 1-5. http://dx.doi.org/10.1016/j.paid.2015.02.011

Byford, J. (2011). Conspiracy theories: A critical introduction. New York: Palgrave Macmillan.

Byford, J. (2014). Beyond belief: The social psychology of conspiracy theories and the study of ideology. In C. Antaki \& S. Condor (Eds.), Rhetoric, ideology and social psychology: Essays in honour of Michael Billig (pp. 83-94). London: Routledge.

Camargo, B. V. (2005). ALCESTE: um programa informático de análise quantitativa de dados textuais. Perspectivas Teórico-Metodológicas em Representações Sociais, 1(3), 511-539.

Camargo, B. V., \& Justo, A. M. (2013). IRAMUTEQ: um software gratuito para análise de dados textuais. Temas em Psicologia, 21(2), 513-518. http://dx. doi.org/10.9788/TP2013.2-16

Douglas, K. M., \& Sutton, R. M. (2008). The hidden impact of conspiracy theories: Perceived and actual influence of theories surrounding the death of Princess Diana. The Journal of Social Psychology, 148(2), 210-222. 
Drinkwater, K., Dagnall, N., \& Parker, A. (2012). Reality testing, conspiracy theories, and paranormal beliefs. The Journal of Parapsychology, 76(1), 57-68.

Goertzel, T. (1994). Belief in conspiracy theories. Political Psychology, 15(4), 731-742. http://dx.doi.org/10.2307/3791630

Goertzel, T. (2010). Conspiracy theories in science. EMBO Reports, 11(7), 493-499. http://dx.doi.org/10.1038/ embor.2010.84

Gouveia, V. V. (2013). Teoria funcionalista dos valores humanos: fundamentos, aplicações e perspectivas. São Paulo: Caso do Psicólogo.

Hofstadter, R. (1966). The paranoid style in American politics. In R. Hofstader (Ed.), The paranoid style in American politics and other essays (pp. 3-40). New York: Knopf.

Jolley, D., \& Douglas, K. M. (2014a). The effects of anti-vaccine conspiracy theories on vaccination intentions. PLoS One, 9(2), 898-906. http://dx.doi.org/10.1371/journal.pone.0089177

Jolley, D., \& Douglas, K. M. (2014b). The social consequences of conspiracism: Exposure to conspiracy theories decreases intentions to engage in politics and to reduce one's carbon footprint. British Journal of Psychology, 105(1), 35-56. http://dx.doi.org/10.1111/bjop. 12018

Jolley, D., Douglas, K. M., \& Sutton, R. M. (2017). Blaming a few bad apples to save a threatened barrel: The systemjustifying function of conspiracy theories. Political Psychology, 39(2), 465-478. http://dx.doi.org/10.1111/pops. 12404

Knight, P. (2008). Outrageous conspiracy theories: Popular and official responses to 9/11 in Germany and the United States. New German Critique, 35(1), 165-193. http://dx.doi.org/10.1215/0094033X-2007-024

Kruglanski, A. W. (1987). Blame-placing schemata and attributional research. In C. F. Graumann \& S. Moscovici (Eds.), Changing conceptions of conspiracy (pp. 219-229). New York: Springer.

Leman, P. J., \& Cinnirella, M. (2013). Beliefs in conspiracy theories and the need for cognitive closure. Frontiers in Psychology, 378(4), 1-10. http://dx.doi.org/10.3389/fpsyg.2013.00378

Lobato, E., Mendoza, J., Sims, V., \& Chin, M. (2014). Examining the relationship between conspiracy theories, paranormal beliefs, and pseudoscience acceptance among a university population. Applied Cognitive Psychology, 28(5), 617-625. http://dx.doi.org/10.1002/acp.3042

Moulding, R., Nix-Carnell, S., Schnabel, A., Nedeljkovic, M., Burnside, E. E., Lentini, A. F., \& Mehzabin, N. (2016). Better the devil you know than a world you don't? Intolerance of uncertainty and worldview explanations for belief in conspiracy theories. Personality and Individual Differences, 98, 345-354. http://dx.doi.org/10.1016/j.paid.2016.04.060

Nicolas, L. (2017). As teorias da conspiração como espelho do século: entre a retórica, a sociologia e a história das ideias. Revista Eletrônica de Estudos Integrados em Discurso e Argumentação, 12, 255-279. Recuperado em outubro 23, 2017, de http://periodicos.uesc.br/index.php/eidea/article/viewFile/1325/1087

Oliver, J. E., \& Wood, T. J. (2014). Conspiracy theories and the paranoid style(s) of mass opinion. American Journal of Political Science, 58(4), 952-966. http://dx.doi.org/10.1111/ajps.12084

R Development Core Team (2012). R: A language and environment for statistical computing. Vienna: R Foundation for Statistical Computing.

Ratinaud, P. (2009). IRAMUTEQ: Interface de R pour les analyses multidimensionnelles de textes et de questionnaires [Computer software]. Retrieved October 23, 2017, from http://www. iramuteq. org

Ratinaud, P., \& Marchand, P. (2012). Application de la méthode ALCESTE à de "gros" corpus et stabilité des "mondes lexicaux": Analyse du "Cable-Gate" avec IraMuTeQ. In Actes des 11 eme Journées internationales d'Analyse Statistique des Données Textuelles (pp. 835-844). Récupéré dans Octobre 23, 2017, from http://lexicometrica.univparis3.fr/jadt/ jadt2012/Communications/Ratinaud,\%20Pierre\%20et\%20al.\%20\%20Application\%20de\%20la\%20methode\%20 Alceste.pdf

Sapountzis, A., \& Condor, S. (2013). Conspiracy accounts as intergroup theories: Challenging dominant understandings of social power and political legitimacy. Political Psychology, 34(5), 731-752. http://dx.doi.org/10.1111/pops.12015

Swami, V., \& Coles, R. (2010). The truth is out there: Belief in conspiracy theories. The Psychologist, 23(7), 560-563.

Swami, V., \& Furnham, A. (2012). Examining conspiracist beliefs about the disappearance of Amelia Earhart. The Journal of General Psychology, 139(4), 244-259. http://dx.doi.org/10.1080/00221309.2012.697932

Swami, V., Voracek, M., Stieger, S., Tran, U. S., \& Furnham, A. (2014). Analytic thinking reduces belief in conspiracy theories. Cognition, 133(3), 572-585. http://dx.doi.org/10.1016/j.cognition.2014.08.006

Swami, V., Weis, L., Lay, A., Barron, D., \& Furnham, A. (2016). Associations between belief in conspiracy theories and the maladaptive personality traits of the personality inventory for DSM-5. Psychiatry Research, 236, 86-90. http:// dx.doi.org/10.1016/j.psychres.2015.12.027 
Trifonova, T. (2017). Agência nos thrillers cinematográficos de conspiração. Rumores, 11(22), 59-88.

Van Prooijen, J. W., \& Acker, M. (2015). The influence of control on belief in conspiracy theories: Conceptual and applied extensions. Applied Cognitive Psychology, 29(5), 753-761. http://dx.doi.org/10.1002/acp.3161

Van Prooijen, J. W., \& Jostmann, N. B. (2013). Belief in conspiracy theories: The influence of uncertainty and perceived morality. European Journal of Social Psychology, 43(1), 109-115. http://dx.doi.org/10.1002/ejsp.1922

Van Prooijen, J. W., Krouwel, A. P., \& Pollet, T. V. (2015). Political extremism predicts belief in conspiracy theories. Social Psychological and Personality Science, 6(5), 570-578. http://dx.doi.org/10.1177/1948550614567356

Wagner-Egger, P., Bangerter, A., Gilles, I., Green, E., Rigaud, D., Krings, F., .. \& Clémence, A. (2011). Lay perceptions of collectives at the outbreak of the H1N1 epidemic: Heroes, villains and victims. Public Understanding of Science, 20(4), 461-476. http://dx.doi.org/10.1177/0963662510393605

Recebido: janeiro 26, 2018

Versão final: maio 23, 2018

Aprovado: agosto 17, 2018 